\title{
Challenges to Recruitment of Urban African American Patients with Cancer Pain
}

\author{
Stephanie Myers Schim ${ }^{1 *}$, April Hazard Vallerand ${ }^{1}$, Susan M. Hasenau ${ }^{2}$ and Sheria Grice Robinson $^{1}$ \\ ${ }^{1}$ Wayne State University College of Nursing, Cohn Building, Cass Avenue, Detroit, USA \\ ${ }^{2}$ Madonna University School of Nursing Livonia, MI, USA
}

Received: February 10, 2014; Accepted: April 29, 2014; Published: May 02, 2014

*Corresponding author: Stephanie Myers Schim, Wayne State University College of Nursing, 234 Cohn Building, 5557 Cass Avenue, Detroit, MI 48202 , USA, Tel: (313) 577-5137; E-mail: s.schim@wayne.edu

\begin{abstract}
There are many assumptions about recruitment of African Americans to cancer studies. The population is often characterized as older, low income, with limited education, scarce resources, and distrust of the health care system. Support systems for African Americans are reported to be centered on family and church communities. Observations made during recruitment and enrollments for a longitudinal intervention study with urban African Americans with cancer pain are presented. Majority of the subjects being recruited in this study are well educated, knowledgeable about research and very open to healthcare providers and researchers. Our population is younger than anticipated and struggling with an absence of family, faith, or community supports. They are more connected in terms of phone ownership, but often difficult to contact due to interruptions in phone service, housing insecurity and time demands related to medical care for their cancer. We conclude that familiarity with cultural patterns of a population of interest is necessary, but local and individual assessment is critical to successful recruitment to research.
\end{abstract}

Keywords: Recruitment; African American; Urban; Cancer; Pain; Community

\section{Introduction}

A fundamental issue in quality research is the need to successfully access the population, recruit potential subjects, and enroll eligible participants. When the population of interest is African American, there is ample literature discussing commonly encountered research issues and cultural patterns [1-5]. However, when the focus is on urban African Americans with cancer pain, there is little guidance available from the literature $[6,7]$. Additionally, challenges observed in our own research suggest somewhat different issues with recruitment. The purpose of this paper is to describe some of the expected and unexpected challenges encountered in the recruitment of Midwestern urban African American patients with cancer pain to a longitudinal, community-based intervention study.

The study from which our observations are derived is a large randomized control trial of a coaching intervention to improve function among African Americans with cancer pain. Following IRB approval, we are recruiting adult men and women who report experiencing moderate-to-severe pain. Pain in patients with cancer may be caused by tumor involvement, associated with cancer therapy, or may be due to factors unrelated to their cancer [8-10]. Most patients with cancer rank pain as their most distressing symptom, regardless of the type of disease, the presence of metastasis, or the treatment regimen [11,12]. Our study focuses on the relationship between pain and functional status rather than type or stage of cancer, therefore, all African American patients with cancer related pain are eligible to participate. The coaching intervention is unique in that, it is culturally tailored to our African American community and individualized to the needs of each specific participant. In this ongoing longitudinal study, participants are followed for 12 weeks. This paper reports on patient recruitment issues observed within the 18 months of the study. Comprehensive reporting of study findings will occur when we complete the study protocol.

In approaching research design with traditionally underserved populations, it is tempting to focus on characteristics that distinguish unique aspects of culture. There is growing consensus, however, that culture needs to be defined and addressed in its broadest connotation to include not only race and ethnicity, but also other characteristics that are commonly used to classify people [13]. Such characteristics include gender, sexual orientation, education, socioeconomic status, employment, values, beliefs, religion/spirituality, and many more $[14,15]$.One classic definition of culture is the learned, shared, and transmitted values, beliefs, norms, and ways of life that guide thinking, decisions and actions in patterned ways [16]. Culture is acquired through processes of growing up or living in a particular place at a specific time in history [17]. Each human being has a way of being in the world that reflects both common and unique aspects of culture and each is certain that their way of being is the correct way. It is not until people encounter and interact with others who have a different "right way" to express culture that the potential for conflict and opportunities for appreciating diversity arise $[13,18]$.

Using standard descriptions of group characteristics, needs, and desires can provide some guidance regarding ways to 
approach a population. For example, when doing research in an African American community, it is widely reported that issues of distrust are common and must be addressed [2,19-22]. Whereas, the overall patterns in a population help to distinguish one group from another, the within-group variation is often observed to be greater than the between-group variation. For example, within African American communities there are many people who do trust researchers [6]. Both group-level knowledge and individual assessment are needed to adequately understand the needs, preferences, and adaptations necessary to conduct research on any population group [18]. Group-level knowledge, aka "holding knowledge," is useful as a way to guide small group and individual assessment [17]. Knowing that trust is an issue for many African Americans, for example, should sensitize investigators to the need to assess the specific people with whom they conduct research for levels of trust and to design procedures to address trust concerns when they are identified. Knowledge of cultural patterns should also guide researchers to never assume that any individual's ways of being in the world are consistent with even their own cultural group patterns.

\section{Observations from Our Experience}

\section{Accessing patients and caregivers}

The specific population of interest for our research is urban African Americans with cancer pain. Patients are being identified through a comprehensive cancer center in a large city. Knowing the population is essential to understanding the people you hope to recruit from any population. Although the literature may describe characteristics of a given group, understanding the specifics of the group in a particular setting and within a given context cannot be done without insider (emic) knowledge [17]. One way to acquire such knowledge is to include on the research team people from the community and those who have extensive experience with community members. In addition, researchers need sustained engagement with a community to grasp the nuances and range of values and beliefs of potential participants. A third and most common way to acquire knowledge is to review the extant literature from those working with similar populations. In our work we are utilizing all three approaches.

Researchers are often exposed to the idea that as a result of historically harmful projects, such as the Tuskegee Syphilis Study [2], African Americans distrust researchers and are therefore generally resistant to participation [4]. Our experience has demonstrated that many African American cancer patients with pain express both trust and interest in research. In preparation for our current study, we conducted a focus group with nine African American patients drawn from the population of interest to assess their current methods of coping with pain, their perceptions of proposed study tools, and their impressions of the planned intervention. Within this small group there was marked enthusiasm for the study and all of the focus group participants indicated that they were eager to enroll. As we moved forward with recruitment for the main study, we observed that many African Americans that we approached were already very knowledgeable about cancer research and their participation options.
It is widely assumed that African Americans living in old inner-city neighborhoods are uneducated, live in poverty, and are underinsured or uninsured [23,24]. Although, this undoubtedly describes many people, we are finding that the people we approach to assess eligibility and interest in research participation actually represent a broad spectrum of education and socioeconomic levels, including patients with advanced education and substantial means. Because, we are accessing the population through a comprehensive cancer center, we usually find that African American patients we approach have some form of health insurance. However, many patients are underinsured and are not be able to get all the recommended medications and treatments. Also, unfortunately, many people we approach have more limited financial resources. Consistent with the literature, we are finding a large number of participants living in urban neighborhoods of boarded-up or burned-out houses. Some families own their homes, but are unable to afford repairs, and have no option to move. Others move frequently, due to personal, social, and economic pressures that cause housing instability and uncertainty.

Family is reported to be a central value among African Americans, with several types of extended or interconnected families being common [25]. We are recruiting dyads of cancer patients and their caregivers. We ask participants to identify as their caregiver the person who helps them the most. In many instances, we are finding that potential participants cannot identify any caregiver at all, while others name multiple people intermittently involved with their care. These caregivers are rarely spouses or partners; sometimes children, siblings or parents; and more often neighbors, friends, or paid helpers. Frequently, the identified caregivers do not live with the patient and are not available to the patient or the researchers because they work full time or have competing responsibilities. It cannot be assumed that even those with extended family in the area can rely on family members for care giving. For myriad reasons, many urban African Americans with cancer find themselves essentially alone.

Another value frequently cited in the literature regarding African Americans is the importance of and reliance on church communities [25]. Churches are central institutions that provide many types of spiritual, social, and instrumental support in the African American community. Based on the literature, we expected to see caregivers and social networks arising from church communities. Although some participants make every effort to attend services when they feel well enough, support from the local church communities has not been widely evident in our recruitment experience. The degree of social isolation observed among African American patients with cancer pain in our area appears to be significant.

The unexpected pattern, of lack of social support for the cancer patients, that became evident during the course of the study, may be attributed to the age distribution pattern of the subjects involved. Potential participants so far seem to be quite a bit younger, than we anticipated. According to the American Cancer Society, the median age of diagnosis for all cancers in all 
races is 66 years [26]. Our first participants $(n=131)$ have a mean age of 53 years, with a range of 22 to 75 years. The generally younger cancer patient age may be reflected in generational differences in family structure and social support with younger people having fewer social and familial networks.

\section{Recruiting participants}

As initially conceptualized, our recruitment plan involved identifying potential participants through referral from ambulatory care clinicians in a large regional cancer center. Although a number of clinicians wrote letters of support suggesting that they would refer patients to the study, we quickly identified a barrier with clinician referral. At any given time there are dozens of clinical trials and behavioral research projects seeking participants from the ambulatory clinics of the comprehensive cancer center. In spite of excellent intentions, the capacity to provide adequate numbers of referrals is limited due to time and attention constraints as well as other organizational issues. These barriers have been identified elsewhere $[27,28]$.

In order to identify sufficient potential participants, we hired several graduate and undergraduate students to spend time in the clinic waiting room. They approach people who appear to be African American, introduce the study, and collect contact information from those indicating willingness to participate. A preference for a face-to-face approach with minority populations has been recently echoed in the literature [5]. Although this recruitment method has yielded sufficient participants for our current study, we have identified three areas of concern using this approach.

One concern is the need to have recruiters present during clinic hours running from early morning to late afternoon, five days a week. As the recruiter positions were not initially budgeted, funding adequate coverage has required creative reallocation of funds and careful scheduling as well as reliance on students in recruiter roles. While a recruiter talks with one potential participant, others are leaving the clinic area and may be missed. Dignan and colleagues [6] identified similar concerns in recruiting African American cancer patients in southern states. Ideally, having at least two recruiters on site together each day would allow more efficient recruitment if such coverage could be funded.

A difficulty of the on-site face-to-face recruitment process is that our team members need to quickly identify who is African American. Because the range of appearance of those who selfidentify as African American or Black is extensive, errors can be made $[29,30]$. Therefore, our team members ask people they approach how they self-identify with regard to race/ethnicity to assess eligibility for the study. The second concern happens occasionally when potential participants ask why only African American patients are eligible and question whether the racial emphasis is discriminatory. When we explain that our focus is on African Americans because of observed differences in pain experiences and perceived control over pain between African Americans and Caucasians [31], people say that this rationale is understandable and acceptable.
The third concern relates to cultural concordance. Racial/ ethnic matching of recruiters and potential participants has been suggested as a way to enhance minority participation in research [5,32]. When we seized an opportunity to add African American recruiters to our team, we noticed some additional issues. One recruiter is a $\mathrm{PhD}$ candidate with extensive nursing experience. Another recruiter is an undergraduate pre-nursing student. Potential participants respond quite positively to the more experienced recruiter. However, although responses to the less experienced recruiter are friendly, people seem to take the offer of participation less seriously. We are addressing this issue through additional training and on-site mentoring support by the more experienced recruiter. Based on our experiences with these two African American team members and several Caucasian recruiters, we conclude that whereas cultural concordance racial/ ethnic matching may be desirable, other characteristics such as maturity, experience, and personality may be just as important. This is consistent with the patterns observed in the literature with regard to preferences for racial concordance [33,34].

Monetary incentives have been identified as a major asset to participant recruitment with the African American population $[33,35]$. In our study, we offer gift cards at the beginning and end of the study for each patient and caregiver. Despite our knowledge that there are many opportunities for participation in research studies through the cancer center, we were surprised when caregivers declined in favor of "studies that pay more." Patients and caregivers recruited from a regional cancer center are research savvy; they have demonstrated knowledge of currently available studies and the competitive monetary incentives offered.

\section{Enrolling participants}

Once people indicate willingness to consider participation and provide contact information to a recruiter, a member of the research team attempts to call them to verify the accuracy of the contact information, to answer additional questions, and confirm willingness to participate. The process of contacting the potential participant by phone for confirmation is essential, but problematic. The initial challenge is reaching the potential participant. The number of calls needed to reach patients is often extensive. Whereas other researchers have limited call attempts to six in a two-week period [7], we find that contact often requires ten or more attempts over several weeks to get someone to answer the phone. Our participants tell us that they don't answer for a variety of reasons including not feeling well enough to talk, not being able to reach the ringing phone and being away from home for doctor appointments and cancer treatments. Early in our enrollment effort, when a phone rang persistently but was not answered, we wondered if people were simply screening their calls and were not answering for phone numbers they did not recognize. After we talked with several of our participants and their family members, we were told that such call screening was not their general practice. In fact, people assured us that, because they are called by a variety of healthcare providers related to their diagnoses and treatments, they routinely try to answer all calls even when the number is unrecognized. 
The fact that caregivers are frequently not living with patients also complicates making connections. Although a message is left if a voice mail system is in place, very rarely are calls returned. Recognizing that attempts to contact are usually limited by restrictions on staff time and energy, we find that among urban African American cancer patients with pain, additional attempts result in increased participant enrollment, thereby justifying the effort. We have also recently identified text messaging as a productive way of contacting some people and are increasing use of this method of communication. We encourage our team callers to be patient and persistent with the realization that enrollment in our study is probably not our participants' top priority. In a recent systematic review of the literature on factors influencing African American enrollment in cancer trials [36], life and environmental stressor were identified.

Other issues related to telephone contacts also challenge enrollment. Frequently our calls are met with an outgoing message that the "phone is no longer in service." We have come to understand with our population that this can mean that the phone has actually been disconnected because the patient has died or moved, but more often it is due to non-payment of the phone bill. We have learned that when someone pays the outstanding balance on the bill, the phone service quickly resumes. Often service is restored within a day or two of our "disconnected" call. Therefore we continue to call those with "no longer in service" messages in the hopes of eventually making contact. Although this additional time and effort is costly, in our population it has proved to be effective in contacting and confirming participants.

As we recruit potential participants, we ask for contact numbers for both the patient and primary caregiver. We also inquire about any additional contact information that we might be able to use to reach them. Frequently, we need to use all of the available contact numbers to reach either the patient or someone who knows the patient's status and whereabouts. In our population of urban African Americans with cancer pain, we observe that many people have landlines and several personal cell phones, but the available phones are not consistently in working order.

The prevalence of multiple phones in households came as a surprise in our study. In fact, expecting that phone access would be a problem in our sample [34], we arranged to provide disposable cell phones with pre-paid minutes as an additional incentive for participation in our study. It is essential for participants to be contactable by phone and to be able to contact the research nurses. We predicted that because a large percentage of our population of interest would be low income urban dwellers, they would have limited access to phones. Although other researchers had observed that most urban African Americans had cell phones we were concerned that many would be reticent to use their minutes for the research project. Purchasing disposable cell phones in bulk presented additional unanticipated challenges. Many cell phone retailers limit purchases to two cell phones per person. Using our university's contacts and many hours of negotiation, we were eventually able to purchase batches of cell phones for study use. During the first year of enrollment, about half of our participants declined the offer of a free study phone in favor of using their existing phones. For those who choose to use their own phones, we add an extra gift card to compensate them for minutes used for the study.

As our initial study design was developed, the provision of cell phones was challenged by some reviewers. Their concerns related to the potential for "misuse" of the provided cell phones for non-study-related calls. We felt that putting restrictions on phone use would communicate a lack of trust to participants. With the community recruitment challenges and longitudinal design of our study, establishment and maintenance of trust is essential. Very few of our enrollees have requested additional minutes beyond what came with their cell phones and the few who asked have been given additional minutes.

\section{Conclusion}

We have described some of the challenges we have experienced in recruiting urban African Americans with cancer pain for a research project. As we considered issues related to accessing the population, recruiting potential participants, and enrolling patients and caregivers in our study, we identified characteristics that were consistent with the literature and characteristics that seem unique to our specific population. As anticipated, many of our potential participants are older, low income, urban African Americans with limited education, scarce resources, and distrust of the health care system. However, many people we recruit are well educated, quite knowledgeable about research, and very open to healthcare providers and researchers. Our population so far is younger than anticipated and struggling with an absence of family, faith, or community supports. They are more connected in terms of phone ownership, but often difficult to contact due to interruptions in phone service, housing in security, and time demands related to medical care for their cancer. Although often challenging to recruit, contact, and enroll in our project, we found that patience and persistence yield participants who not only enroll, but enthusiastically complete the 12-week longitudinal study. To successfully recruit urban African American patients with cancer pain, our experience demonstrates the need to balance general knowledge of the population with careful local and individual assessment and adjustment of recruiting strategies based on specific participant and urban community needs.

\section{Acknowledgement}

The authors wish to thank our grant consultants for their careful review and insightful feedback on this manuscript: Ms. Linda Vanni (St. John Health System, Detroit, MI), Dr. Lisa Campbell (East Carolina University, Greenville, NC), and Dr. Tamara Baker (University of South Florida, Tampa, FL.)

\section{Funding/Support}

This work was supported in part by NCI grant \# 1 R01 CA149432-01A1(Dr. Vallerand).

\section{References}

1. Shavers VL, Lynch CF, Burmeister LF (2002) Racial differences in factors 
that influence the willingness to participate in medical research studies. Ann Epidemiol 12(4): 248-256.

2. Scharff DP, Mathews KJ, Jackson P, Hoffsuemmer J, Martin E, et al. (2010) More than Tuskegee: understanding mistrust about research participation. J Health Care Poor Underserved 21(3): 879-897.

3. Kneipp SM, Lutz BJ, Means D (2009) Reasons for enrollment, the informed consent process, and trust among low-income women participating in a community-based participatory research study. Public Health Nurs 26(4): 362-369.

4. Shellman J, Mokel M (2010) Overcoming barriers to conducting an intervention study of depression in an older African American population. J Transcult Nurs 21(4): 361-369.

5. George S, Duran N, Norris K (2014) A systematic review of barriers and facilitators to minority research participation among African Americans, Latinos, Asian Americans, and Pacific Islanders. Am J Public Health 104(2): 16-31.

6. Dignan M, Evans M, Kratt P, Pollack LA, Pisu M et.al., (2011) Recruitment of low income, predominantly minority cancer survivors to a randomized trial of the I Can Cope cancer education program. J Health Care Poor Underserved 22(3): 912-924.

7. Northouse LL, Rosset T, Phillips L, Mood D, Schafenacker A et al., (2006) Research with families facing cancer: the challenges of accrual and retention. Res Nurs Health 29(3): 199-211.

8. Bruera E, Kim HN (2003) Cancer pain. JAMA 290(18): 2476-2479.

9. Foley KM (1979) Pain syndromes in patients with cancer, in Advanced Pain Research and Therapy. In: Bonica JJ \& Ventafridda V (Eds.), Raven Press, San Diego, California.

10. McGuire DB (1995) The multiple dimensions of cancer pain: A framework for assessment and management. In: McGuire DB, Yarbo CH, Ferrell BR (Eds.), Cancer Pain Management. Jones \& Bartlett Boston, pp. 1-17.

11. Wells N, Murphy B, Wujcik D, Johnson R (2003) Pain-related distress and interference with daily life of ambulatory patients with cancer with pain. Oncol Nurs Forum 30(6): 977-986.

12. Clotfelter CE (1999) The effect of an educational intervention on decreasing pain intensity in elderly people with cancer. Oncol Nurs Forum 26(1): 27-33

13. Schim SM1, Doorenbos A, Benkert R, Miller J (2007) Culturally congruent care: putting the puzzle together. J Transcult Nurs 18(2): 103-110.

14. Edberg M (2013) Essentials of Health, Culture and Diversity: Understanding People, Reducing Disparities. In: Riegelman R (Ed.), Essential Public Health. Burlington MA: Jones \& Bartlett.

15. Purnell LD (2013) Transcultural Health Care: A Culturally Competent Approach. In: Davis FA (Ed.), ( $4^{\text {th }}$ edn), Philadelphia.

16. Leininger MM (1997) Overview of the theory of culture care with the ethnonursing method. J Transcult Nurs 8(2): 32 - 52.

17. Leininger MM, McFarland MR (2006) Culture Care Diversity and Universality: A Worldwide Nursing Theory. ( $2^{\text {nd }}$ edn $)$, Sudbury, MA: Jones \& Bartlett

18. Schim SM, Doorenbos AZ (2010) A three-dimensional model of cultural congruence: framework for intervention. J Soc Work End Life Palliat Care 6(3-4): 256-70.

19. Benkert R, Peters RM, Clark R, Keves-Foster K (2006)Effects of perceived racism, cultural mistrust and trust in providers on satisfaction with care. J Natl Med Assoc 98(9):1532-40.

20. Durant RW, Dr Bruce E. Landon (2011)Different types of distrust in clinical research among whites and African Americans. J Natl Med Assoc 103(2):123-30.

21. Russell E,Robinson DH, Thompson NJ, Perryman JP, Arriola KR(2012) Distrust in the healthcare system and organ donation intentions among African Americans. J Community Health 37(1): 40-7.

22. Sims CM, Ethnic notions and healthy paranoias (2010): understanding of the context of experience and interpretations of healthcare encounters among older Black women. Ethn Health, 15(5): p. 495-514.

23. Martin MY et al (2011) Tailoring cancer education and support programs for low-income, primarily African American cancer survivors. Oncol Nurs Forum. 38(1): pg E55-9.

24. Spector RE (2013) Cultrual Diversity in Health and Illness. 8th ed Boston: Pearson.

25.25. Campinha-Bacote J(2013) People of African American Heritage, in Transcultural Health Care: A Culturally Competent Approach LD Purnell Editor F A Davis: Philadelphia.

26. American Cancer Society, Cancer facts \& figures for African Americans 2012-2013, 2013: Atlanta.

27. Joseph G, Dohan D (2009)Recruiting minorities where they receive care: Institutional barriers to cancer clinical trials recruitment in a safety-net hospital. Contemp Clin Trials 30(6): p. 552-559.

28. ?.Donovan JL, Paramasivan S, de Salis I, Toerien M (2014)Clear obstacles and hidden challenges: understanding recruiter perspectives in six pragmatic randomised controlled trials. Trials, 15:5.

29. Smith S(2012)"What are you?Racial ambiguity and the social contruction of race in the U.S in Sociology University of North Texas.

30. Kahn KB ,PG Davies(2011) Differentially dangerous? Phenotypic racial stereotypicality increases implicit bias among ingroup and outgroup members. Group Processes \& Intergroup Relations,. 14(4): p. 569-580.

31. Vallerand AH, Hasenau S, Templin T, Collins-Bohler D(2005)Disparities between black and white patients with cancer pain: the effect of perception of control over pain. Pain Med 6(3): p. 242-50.

32. Johnson VA et al(2011) A systematic review of strategies that increase the recruitment and retention of African American adults in genetic and genomic studies. ABNF J 22(4): p. 84-88.

33. Anderson $\mathrm{KO}$ et al(2002) Cancer pain management among underserved minority outpatients: perceived needs and barriers to optimal control. Cancer 94(8):2295-304.

34. Cleeland CS et al(1994) Pain and its treatment in outpatients with metastatic cancer. N Engl J Med 330(9):592-6.

35. Friedman DB Foster C, Bergeron CD, Tanner A, Kim SH (2014) A Qualitative Study of Recruitment Barriers, Motivators, and CommunityBased Strategies for Increasing Clinical Trials Participation Among Rural and Urban Populations. Am J Health Promot

36. Rivers D,August EM, Sehovic I, Lee Green B, Quinn GP(2013) A systematic review of the factors influencing African Americans' participation in cancer clinical trials. Contemp Clin Trials 35(2):13-32. 